The $[E]$ matrix referred to earlier in the paper is as follows:

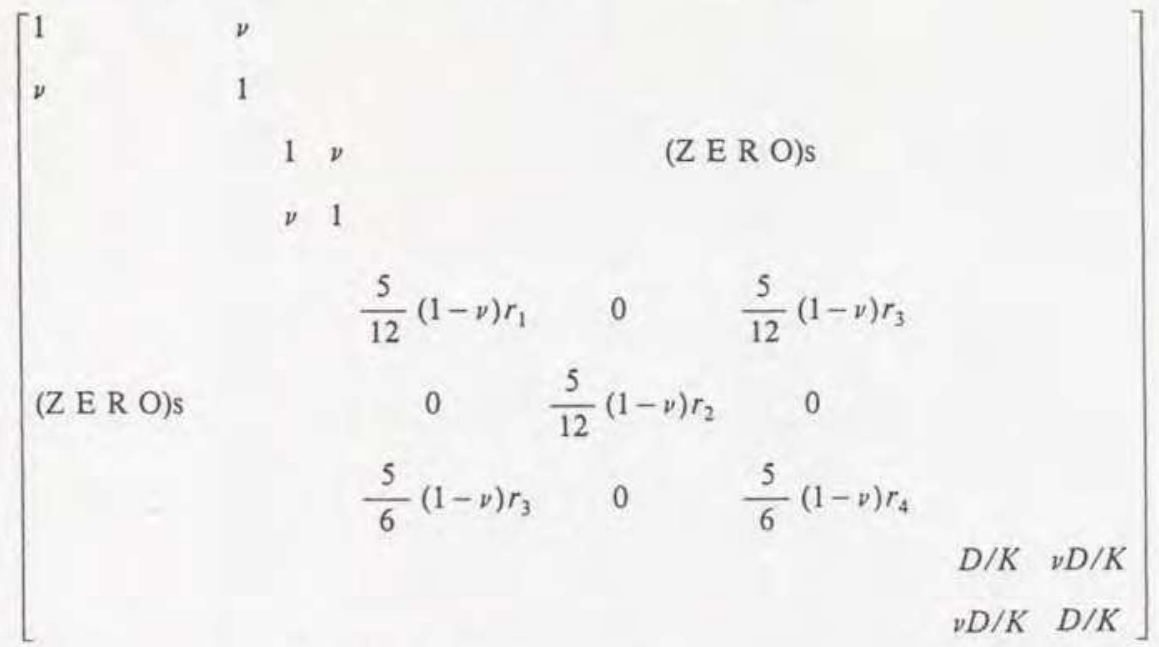

where $K=\mathrm{Eh} /\left[\left(1-\nu^{2}\right)\right]$ and $D=\mathrm{Eh}^{3} /\left[12\left(1-\nu^{2}\right)\right]$

\section{Large-Amplitude Sloshing With Submerged Blocks}

\section{A. Huerta ${ }^{4}$ and W. K. Liu ${ }^{5}$}

The computer simulation of forced vibrations induced on a water pool is presented in this paper. The complexity of the seismic fluid-structure interaction problem is accentuated by the large free surface motion. To overcome this difficulty, the arbitrary Lagrangian Eulerian (ALE) finite element formulation is employed. Moreover, the nonlinear behavior of the free surface motion is also taken into account. The results of the numerical simulation are compared with published experimental data and the effectiveness of the ALE algorithm is demonstrated.

\section{Introduction}

Considerable research in vibration and seismic analysis for various fluid-structure systems have been carried out in the past two decades. Most of the approaches are formulated within the framework of finite elements, and the majority of the work deals with inviscid fluids. However, the application of finite element analysis to viscous flows with large free surface motion is still rudimentary. This paper describes the application of the arbitrary Lagrangian Eulerian (ALE) mixed formulation, see Huerta and Liu (1988), to evaluate the sloshing response of a two-dimensional idealization of a spent fuel storage tank of nuclear facilities.

A particular feature of these tanks is the presence of submerged storage ranks that are usually modelled by under water rigid blocks, Muto et al. (1985). Solving the field equations, i.e. momentum and continuity, for incompressible fluids at medium to large Reynolds numbers, in a fluid domain which has a moving boundary (free surface) and fixed boundaries (rigid blocks), requires an involved kinematic description.

\footnotetext{
${ }^{4}$ Departamento de Matemática Aplicada II, Escuela Téenica Superior de Ingenieros de Caminos, Canales y Puertos, Universitat Politécnica de Catalunya, Barcelona, Spain

5Department of Mechanical Engineering, The Technical Institute, Northwestern University, Evanston, IL 60208, Mem. ASME

Contributed by the Pressure Vessels and Piping Division and presented at the Pressure Vessels and Piping Conference, Honolulu, Hawaii, July 23-27, 1989. of THE AMERICAN SOCIETY of MECHanical EngineERs. Manuscript received by the PVP Division, April 18, 1989; revised manuscript received September 14. 1989.
}

Two classical descriptions are usually used in continuum mechanics. The first is Lagrangian, in which the mesh points coincide with the material particles. In this description, no convective effects appear, and this simplifies considerably the numerical calculations; moveover, a precise definition of the moving boundaries and interfaces is obtained. However, the Lagrangian description does not handle satisfactorily the material distortions that lead to element entanglement. On the other hand, the second description is the Eulerian viewpoint, which allows strong distortions without problems because the mesh is fixed with respect to the laboratory frame and the fluid moves through it. However, this approach presents two important drawbacks (i) convective effects, which introduce numerical difficulties, arise due to the relative movement between the grid and the particles; and (ii) sophisticated mathematical mappings between the grid and the particles are required to follow the interface movement and they often lead to inaccuracy.

Because of the shortcoming of purely Lagrangian and Eulerian descriptions, arbitrary Lagrangian-Eulerian techniques were developed, first in finite differences, Noh (1964) and Pracht (1975), among others; and then in finite elements, Belytschko et al. (1980), Donea (1983), Huerta and Liu (1988), and Liu et al. (1988), among others. This approach is based on the arbitrary movement of the reference frame, which is continuously rezoned in order to allow a precise description of the moving interface and to maintain the element shape.

It should be noticed that the presence of submerged rigid blocks increases numerical difficulties. The submerged blocks impose an Eulerian description in their vicinity in contrast with the vertical Lagrangian description of the free surface. These requirements can be fulfilled by the ALE formulation: (i) at the free surface the mesh follows the vertical movement of the surface and remains fixed horizontally, (ii) around the blocks the mesh is fixed, and (iii) in between, a transition zone is defined with an arbitrary mesh motion that maintains the regularity of the element shape. Moreover, the blocks disturb the flow path and generate recirculations, principally at medium to large Reynolds numbers. This causes numerical difficulty and it can be overcome, for example, using a streamline upwind Petrov-Galerkin formulation, see Brooks and Hughes (1982).

In the next section, the governing equations and a dimensional analysis are presented. Then, the numerical results are discussed and compared with experimental test results. Finally, the concluding remarks are presented. 


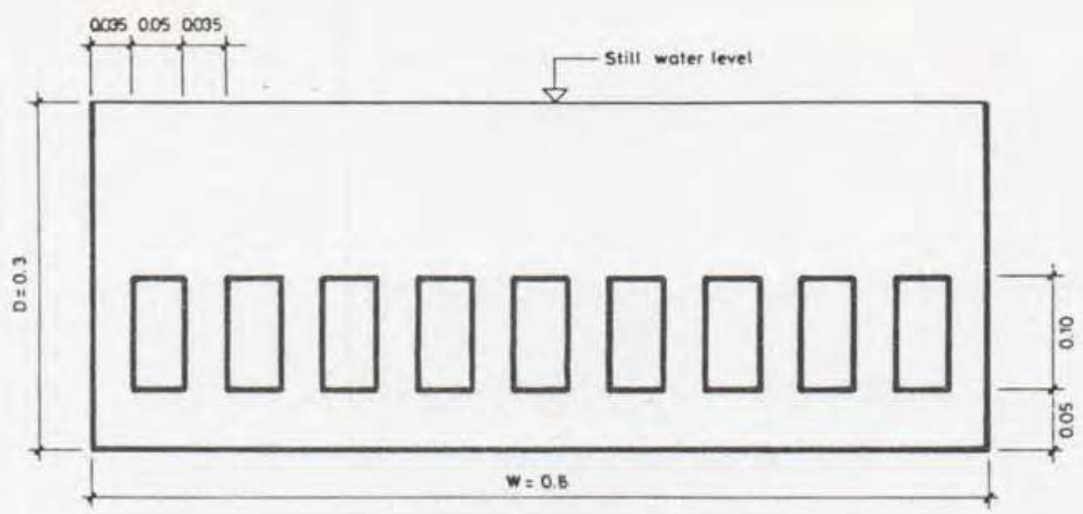

Fig. 1 Two-dimensional model configuration

\section{Dimensional Analysis}

The problem under study is completely determined once (i) the geometry, (ii) the fluid constants, (iii) the external excitation, and (iv) the equations that must be satisfied, are defined. In order to reduce the number of parameters which govern the problem, the dimensionless problem is first to be studied. The problem statement is given in Fig. 1. The twodimensional rigid container has a depth $D$ and a width $W$, the dimensions of the submerged blocks are defined in Fig. 1. The fluid is assumed Newtonian, hence, only density, $\rho$, and viscosity, $\mu$, areneeded. The body forces are those due to gravity
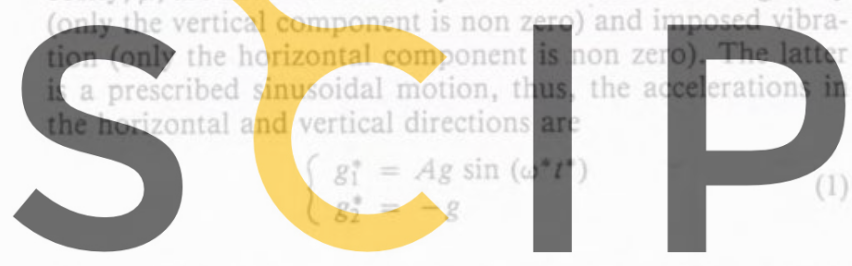

where $g, \omega^{*}$, and $t^{*}$ are the acceleration of gravity, circular Register for free at https//Wwwiscipediar com tion. In this section super-star (") stands for dimensioned variables, lower subscripts denote the components of a tensor, and repeated indices imply sumation over the number of spatial dimensions. The continuity and equilibrium equations which must be satisfied, under an ALE formulation, Huerta and Liu (1988), are written as

$$
\frac{\partial v_{i}^{*}}{\partial x_{i}^{*}}=0
$$

$\rho \frac{\partial v_{i}^{*}}{\partial t^{*}}+\rho c_{j}^{*} \frac{\partial v_{i}^{*}}{\partial x_{j}^{*}}=-\frac{\partial P^{*}}{\partial x_{i}^{*}}+\mu \frac{\partial}{\partial x_{j}^{*}}\left(\frac{\partial v_{i}^{*}}{\partial x_{j}^{*}}+\frac{\partial v_{j}^{*}}{\partial x_{i}^{*}}\right)+\rho g_{i}^{*}$

where $\mathbf{v}^{*}$ is the material velocity, $\mathbf{x}^{*}$ are the spatial coordinates, $P^{*}$ is the pressure, and $\mathbf{c}^{*}$ is the convective velocity which is defined in equations (8).

Once the problem definition is completed, the governing characteristic dimensions are chosen: length $D$, velocity $\sqrt{g D}$, acceleration $g$, pressure $\rho g D$, and time $\sqrt{\frac{D}{g}}$. All the other characteristic dimensions are automatically deduced from these; for instance, the characteristic viscosity and density are, respectively, $\rho \sqrt{g D} D$ and $\rho$. The problem is rewritten in terms of dimensionless variables:

$$
v_{i}=\frac{v_{i}^{*}}{\sqrt{g D}} x_{i}=\frac{x_{i}^{*}}{D}
$$
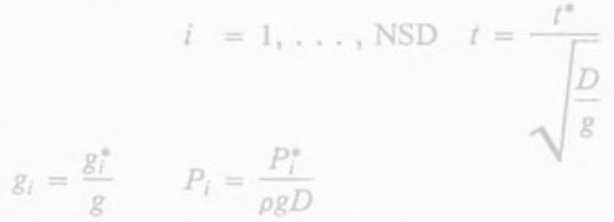

After substitution of equations (3) into equations (2), the following dimensionless equations are obtained:
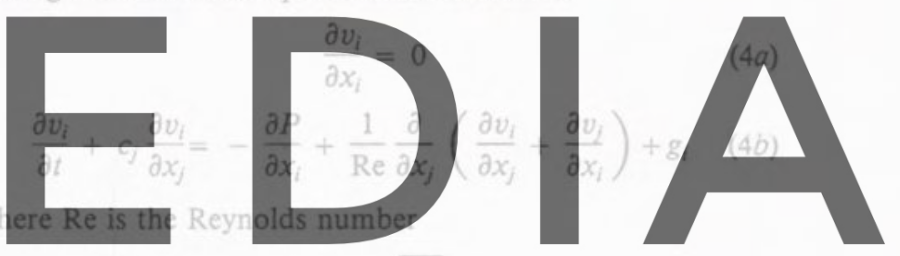

$R_{e}=\sqrt{g D D}$

to download the version without the watermark

The dimensionless external excitation is also obtained wher the scaling definitions, equations (3), are substituted into equation (1); namely,

$$
\left\{\begin{array}{l}
g_{1}=\frac{g_{i}^{*}}{g}=A \sin (\omega t) \\
g_{2}=\frac{g_{2}^{*}}{g}=-1
\end{array}\right.
$$

where $t$ is the dimensionless time defined in equations (3), and the dimensionless circular frequency $\omega$ is

$$
\omega=\omega^{*} \sqrt{\frac{D}{g}}=2 \pi f \sqrt{\frac{D}{g}}
$$

$f$ being the frequency of the excitation.

The dimensionless problem is governed by: all the geometrical data scaled by $D$, the Reynolds number, and the arbitrary parameters of the excitation $A$ and $f$. Notice that the dimensionless fluid constants are the density (1) and the viscosity $(1 / R e)$, and the field equations, equations (4), only depend on the Reynolds number; while the excitation, equation (6), is determined only by the amplitude and the excitation frequency.

In order to complete the definitions, the convective velocity, i.e., c, must be determined. This velocity is, in fact, related to the mesh rezoning algorithm. It is defined as 


$$
c_{i}=\hat{v}_{i}-v_{i}
$$

If the domain boundaries are known at every instant, $\hat{v}$ can be prescribed accordingly. That is, the mesh motion can be described by simple ad-hoc formulas or by solving potential equations that maintain element regularity. If the boundary motion is unknown, that is the case for free surface flows, an extra equation must be solved. This equation is nothing else than the kinematic free surface condition which is a formalization of the physical condition: no particles can cross a material surface (in this case the free surface); more detailed information can be found in Huerta and Liu (1988). The other necessary condition along the free surface (the dynamic condition) states, in this case, that the free surface is a stress free boundary and, as usual, stress conditions on the boundaries are "natural boundary conditions." Therefore, it is automatically included in the weak, or variational form of equations
(4). In this case a mixed remeshing formulation is employed. The kinematic free surface boundary condition is solved along that boundary, while in the interior, the element motion is prescribed as an explicit function of the free surface vertical displacements. This reduces considerably the numerical burden of free surface computations, because remeshing takes place in the domain but the extra equation is only solved along the free surface (smaller than the complete fluid domain).

\section{Numerical Tests}

Model Definition. The goal here is to demonstrate the applicability of the ALE formulation. To illustrate the finite element code, the problem studied experimentally by Muto et al. (1985) is reproduced numerically.

The geometry is given in Fig. 1. The density of the fluid is equal to that of water $\rho=10^{3} \mathrm{Kg} / \mathrm{m}^{3}$, while the values of

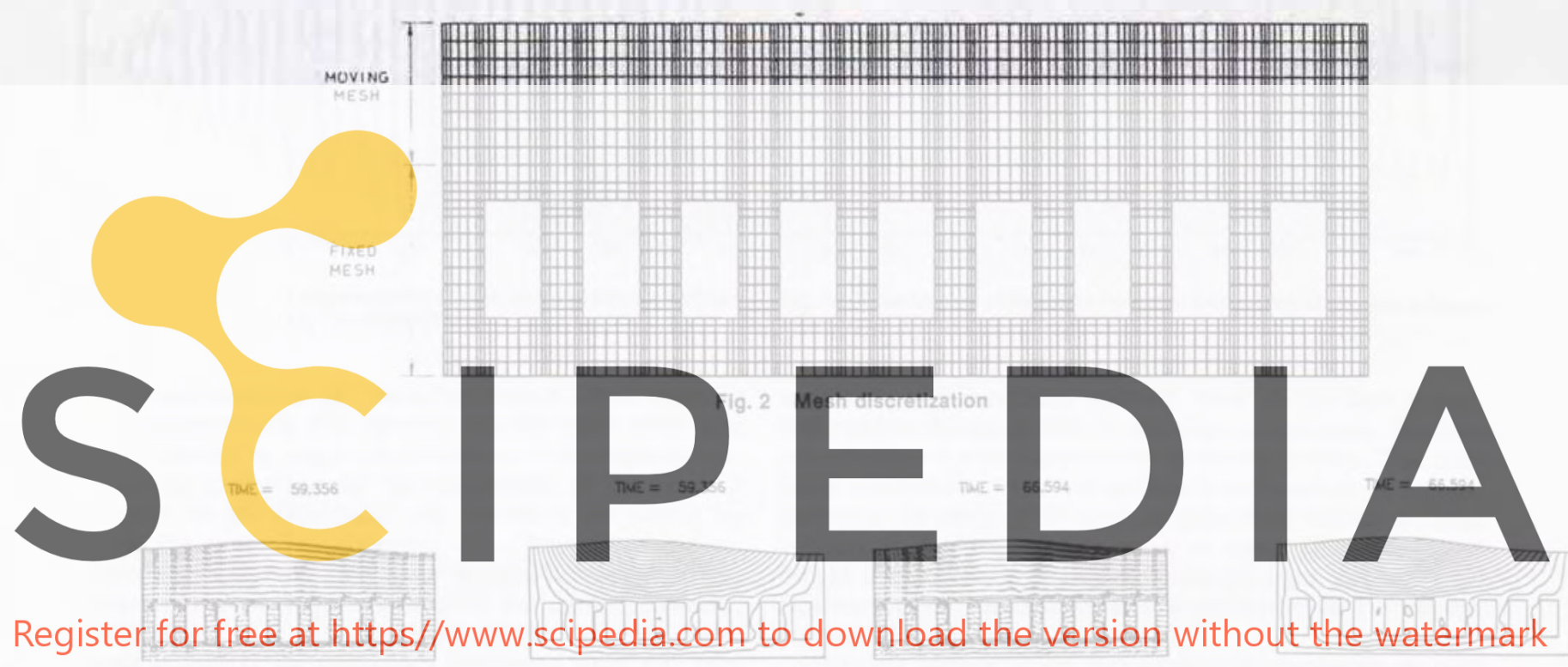

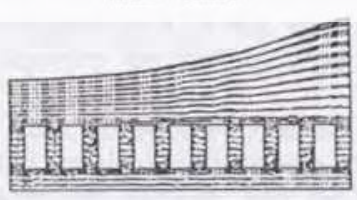

TME $=62.251$

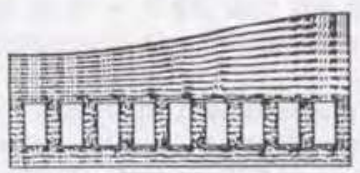

TME $=63.699$

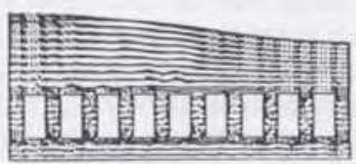

$\mathrm{TME}=65.1 \mathrm{~K}$

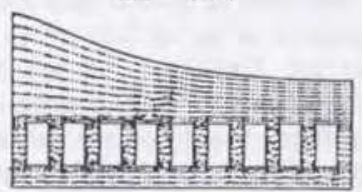

Fig. 3(a) Instantaneous configurations of the domain velocities, and streamlines for the ninth cycle

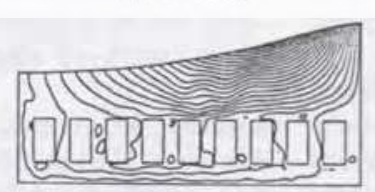

$\mathrm{TME}=62.251$

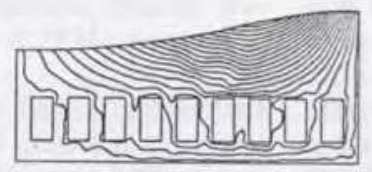

$\mathrm{nME}=63.699$

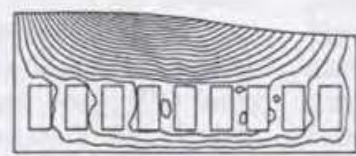

$\mathrm{RME}=65.167$

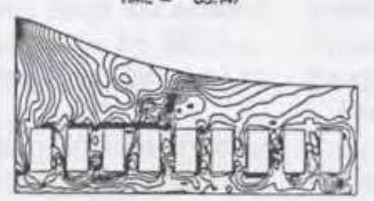

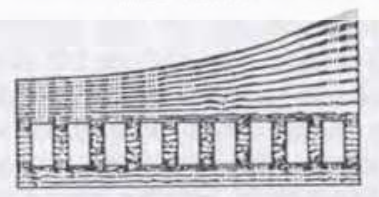

TME $=69.490$

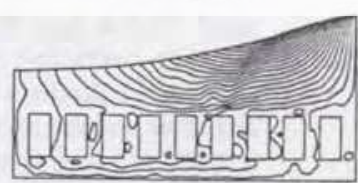

TME $=69.490$

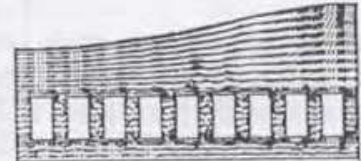

$\operatorname{TME}=70.937$

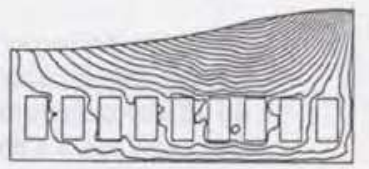

TME $=70.93$

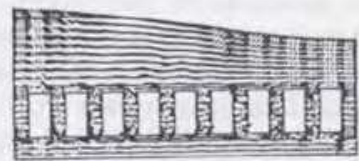

$\mathrm{TME}=72.385$
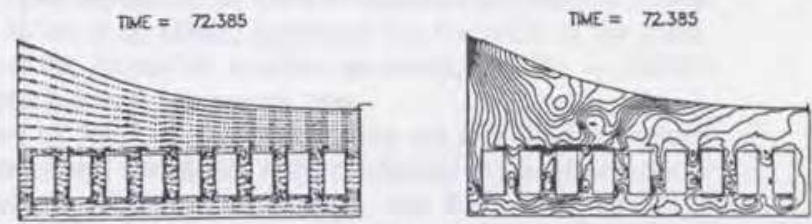

Fig. 3(b) Instantaneous configurations of the domain velocities, and streamlines for tenth cycle 


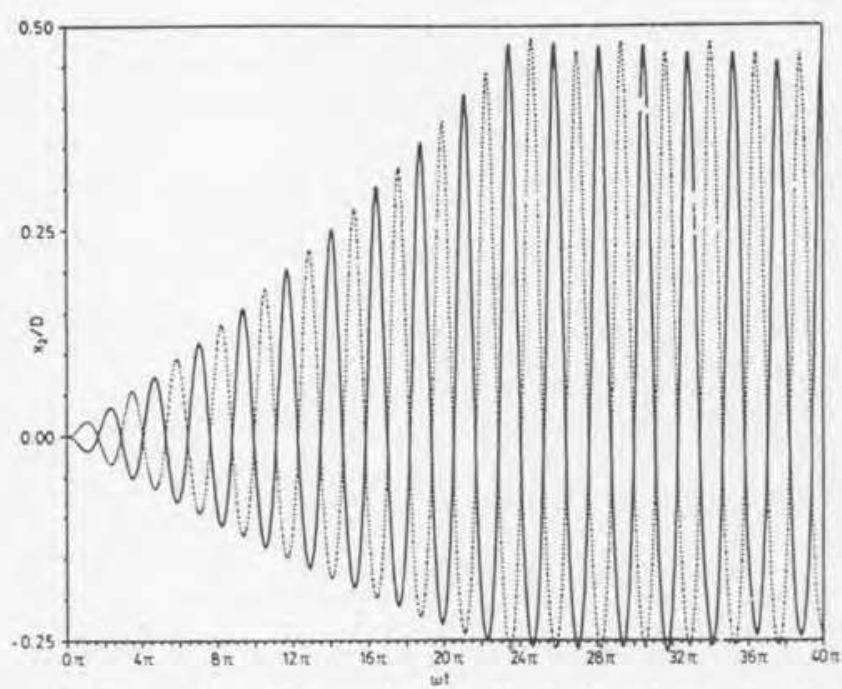

Fig. 4 Time history of the wave heights at both sides of the tank cycles and with submerged blocks, $R e=500000$

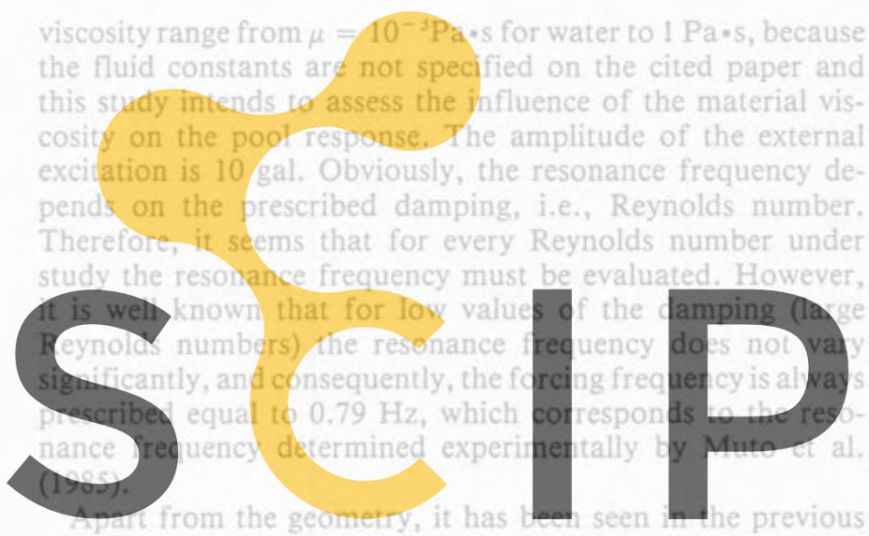

section that the sloshing response of the pool is only governed

Register for free ath https//WwW.scipedia.com

amplitude is $A=0.01$, and the dimensionless circular fre-

(7).

Figure 2 shows the discretization of the fluid domain used for the computations. It consists of 2758 constant pressurebilinear velocity elements ( 25 layers) which imply 3052 nodes. Notice the concentration of elements around the blocks needed to capture recirculation. Two areas are defined in the domain, see Fig. 2; an Eulerian description is implemented in the bottom one (i.e., the mesh is fixed), while the mixed ALE formulation, Huerta and Liu (1988), is used in the upper zone. The latter formulation allows a continuous remeshing such that element shapes are maintained.

The time step chosen is such that 200 time steps are needed in every cycle; that is, $\Delta t$ is obtained dividing $2 \pi$ by $200 \times \omega$. However, for $\mathrm{Re}=500000,300$ time steps must be used in the tenth cycle due to large convection.

Concerning the boundary conditions, zero velocities are prescribed around the blocks, while perfect sliding boundaries are assumed for the bottom and sides of the tank. The material surface conditions described in the previous section are imposed on the free surface.

Numerical Results. The case corresponding to pure water, i.e., $\operatorname{Re}=500000$, is studied first. Instantaneous configurations of the domain with velocity vectors and streamlines are plotted in Fig. 3 for the nineth and tenth cycles. At $\omega t=n \pi$ ( $n$ integer), i.e., at maximum amplitude and minimum velocity, the recirculation around the blocks is obvious. At minimum

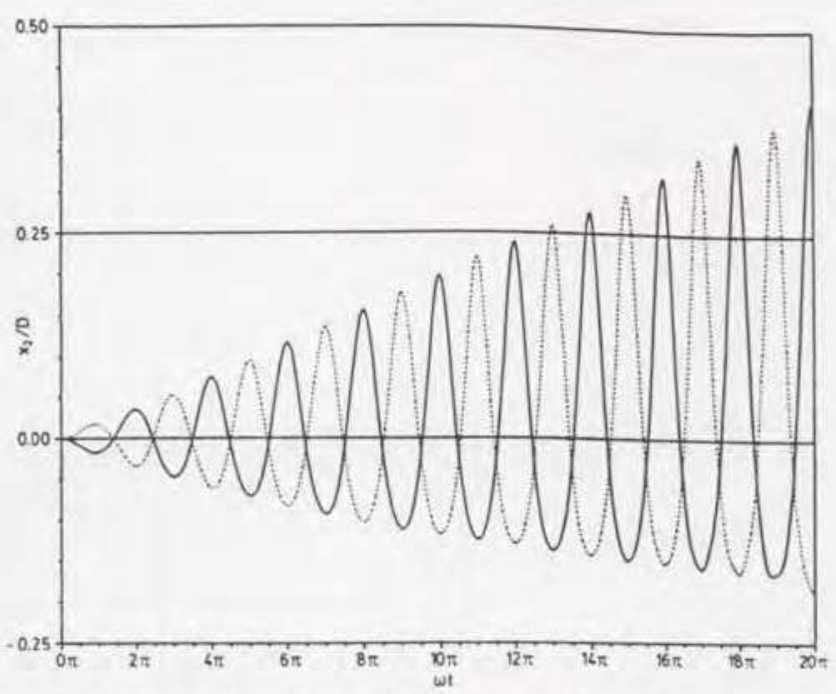

Fig. 5 Time history of the wave height at both sides of the tank without submerged blocks, $\mathrm{Re}=500000$

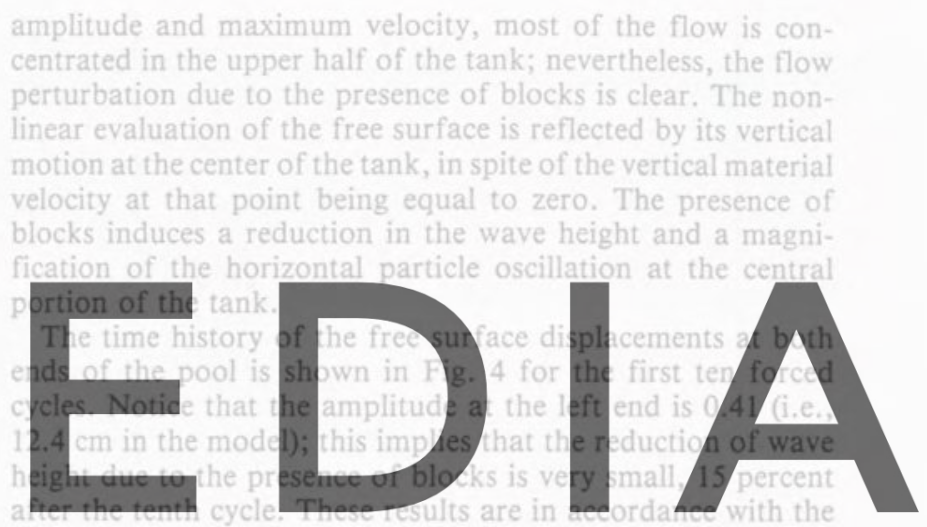

previous ones obtained by the authors, Huerta and Liu (1988),

to download the version without the watermark

only energy losses, see equations (4), are due to the viscous effect. The presence of blocks creates recirculation which increases the viscous losses; however, with such a large Reynolds number the viscous damping is small. This does not agree with the experimental results obtained by Muto et al. (1985). Several possible reasons may be advanced. Apart from the gap between blocks and glass which contradicts the hypothesis of pure twodimensional flow, and the springs subjecting the blocks which create a flow disturbance and allow some movement of the submerged blocks; it is believed, that the loci of conffetti like brass-foil introduced in the fluid to visualize the particle movement by Muto et al. (1985) increased the viscosity of the fluid. Therefore, the Reynolds number assumed, i.e., $\mathrm{Re}=500000$ (pure water), is not the actual one.

Because of the lack of information on the fluid constants (i.e., density and viscosity), a simple sensitivity analysis on the viscous effects is presented; that is, the Reynolds number is prescribed equal to 500 and 5000 . Figure 6 shows the time history of the amplitude at the left end for all the Reynolds numbers. To be consistent with the previous analysis, during the first ten cycles forced vibrations are imposed, while free vibrations are allowed after the tenth cycle for ten more cycles.

The influence of the viscous effects is clearly observed; for $\mathrm{Re}=500$, a " $2 \pi$ steady state" is readily obtained after the first five cycles, and the maximum amplitude is 0.09 (i.e., 2.7 $\mathrm{cm}$ ); this is a reduction of the 80 percent compared to the case of $\mathrm{Re}=500000$. Moreover, the vibrations are clearly damped 


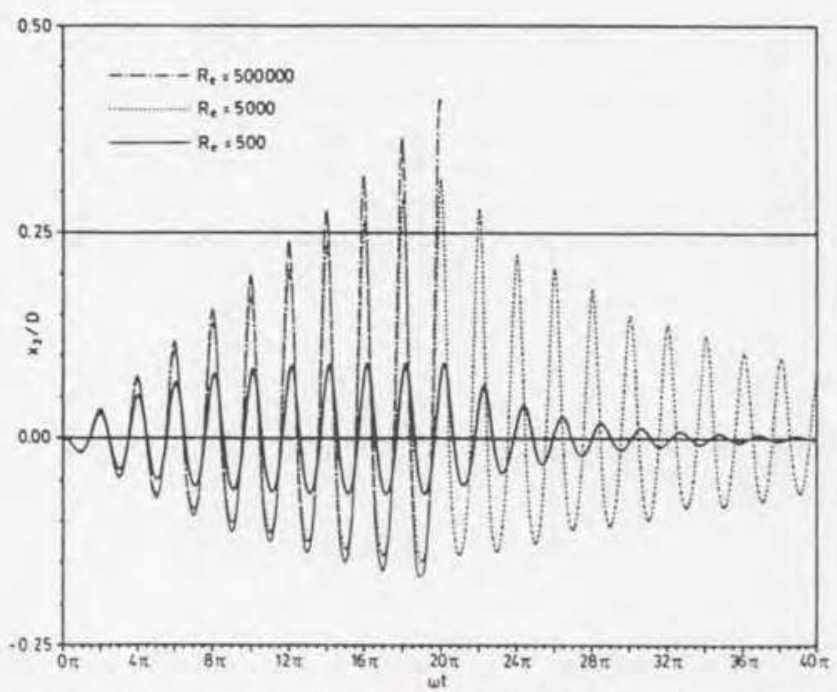

Fig. 6 Time history of the wave height at the left wall with submerged blocks reduces in 15 percent the wave height after ten cycles when $\mathrm{Re}$ $=500000$. If $\mathrm{Re}=5000$ the reduction in wave height is 34 percent, and if $\mathrm{Re}=500$ it is over 80 percent. It seems obvious, after this numerical simulation, that the presence of submerged blocks amplifies the viscous losses because of the recirculation they induce. In the experimental analysis, the loci conffettilike brass-foil introduced in the fluid to observe the particle motion may have increased the viscosity and consequently reduced the wave height. From a practical point of view, this may imply that, if the viscosity is small (large Reynolds number), the presence of blocks alone may not reduce enough the wave height to preclude a potential seismic hazard in spent fuel storage tanks.

\section{Acknowledgments}

The work of Antonio Huerta was supported by the Catalan Government under grants CIRIT AR87 and CIRIT EE88/1. The support of W. K. Liu by the National Science Foundation is also gratefully acknowledged.

\section{References}

Relutcrhkn T Kennedv, I. M. and Schocberie, D. F.. 1980."Ouasi-Eulerian inite Element Formulation for Fluid Structure Interaction," ASME JourNi pF Pressure Vessel. Technology, Vol. 102, pp. 62-69. Brooks, A. N., and Hughes, T. J. R., 1982, "Streamline Upwind/Petroy

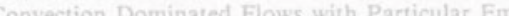
hasis on the Incompressible Navier-Stokes Equations," Computer Methods in 4 pplied Mechanics and Engineering, Vol. 32, pp. 199-259 Donea, I 1983, "Arhitrary Lagrangian-Eulerian Finite Element Methods, Computational Methods for Transient Analysis, eds.. T. Belytschko and T. J . Hughes, Elvesier Science Publishers, pp. 473-516. Anerta. A and Lit, W. K., 1988, "Viscous. Flow with Large Free Surface Gotion." Computer Methods in Applied Mechanics and Engineering, Vol. 69.

For Re $=5000$ no stationary maximum wave amplitude is observed after ten forced cycles. The measured value after the tenth cycle is 0.32 (i.e., $9.6 \mathrm{~cm}$ ) which is already larger than the stationary value observed by Muto et al. (1985), i.e., $6 \mathrm{~cm}$; compared to the results for pure water the reduction is however
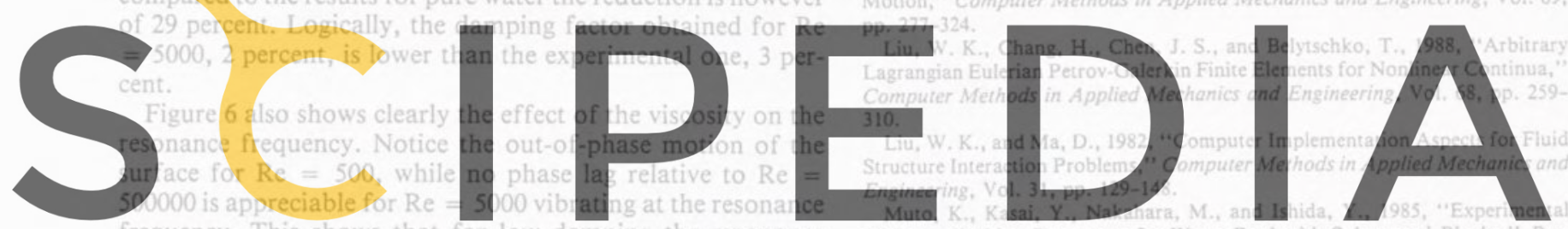

requency. This shows that for low damping the resonance

frequency does not vary considerably and validates the choice

Register forfree at https//www.scipedia.com to download the version without the watermark

\section{Conclusions}

Based on the results described in this study of large amplitude sloshing with submerged blocks, the following conclusions can be advanced:

1 The pressure-velocity mixed formulation and the streamline upwind Petrov-Galerkin techniques are now frequently used methods to compute confined incompressible flows with large Reynolds numbers and strong convective effects. The implementation of the arbitrary Lagrangian-Eulerian formulation into such preexisting codes is simple. And moreover, this formulation allows an efficient and accurate description of large free surface motions.

2 While resonance frequencies are easily obtained by linear codes in sloshing problems, the surface displacements depend strongly on the nonlinearities if large motions occur. The ALE description takes into account the nonlinear effects on both the surface and the interior of the domain.

3 Numerical tests such as the one presented herein reduce considerably the experimental costs and allow, if needed, a complete sensitivity analysis on the material properties or geometry of the problem. That is, numerical simulations which were confined to "qualitative" results, can now be used to obtain "quantitative" results.

4 While in the experimental analysis, the reduction of wave height is only attributed to the presence of blocks. The present analysis shows clearly the combined effect of the submerged blocks and the viscosity of the fluid. The presence of blocks

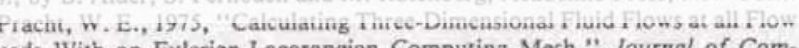
Speeds With an Eulerian-Lagarangian Computing Mesh," Journal of Computational Physics, Vol. 17, pp. 132-159. 